Med Klin Intensivmed Notfmed 2015 · 110:326-327 DOI 10.1007/s00063-015-0038-7

Online publiziert: 20. Mai 2015

(c) Springer-Verlag Berlin Heidelberg 2015

\author{
O. Heinzel ${ }^{1} \cdot$ F. Hoffmann ${ }^{2}$ \\ ${ }^{1}$ Universitätskinderklinik, Tübingen, Deutschland \\ ${ }^{2}$ Kinderklinik und Kinderpoliklinik im Dr. von Haunerschen Kinderspital, München, Deutschland
}

\title{
Der Kindernotfall - eine große Herausforderung
}

noch utopisch, flächendeckend spezialisierte Kindernotarztsysteme zu implementieren, wie sie sich in manchen deutschen Großstädten seit Jahren bewähren. Somit bleibt den in der Notfallmedizin engagierten Nichtpädiatern nur, sich in regelmäßigen Fortbildungen, Workshops und durch die Lektüre von praxisorientierten Publikationen das Handwerkszeug für eine qualitativ hochwertige Versorgung anzueignen. Bei der Erstversorgung eines kritisch kranken Kinds ist es gerade in der Notfallmedizin essenziell, eine symptomorientierte und algorithmenbasierte Behandlung durchzuführen.

\section{》) Die Erstversorgung soll symptomorientiert und algorithmenbasiert erfolgen}

Mittlerweile stehen auch für den pädiatrischen Bereich Hilfsmittel zur Verfügung, die es auch dem Nichtspezialisten ermöglichen, ein Kind adäquat zu versorgen. Dass dies manchmal nicht dem Versorgungsstandard auf einer großen Kinderintensivstation eines pädiatrischen Maximalversorgers genügt, liegt in der Natur der Sache. Dies kann jedoch nicht das Ziel einer pädiatrischen Notfallversorgung in der Breite sein.

In den Leitartikeln dieser Ausgabe werden wichtige Themen der Kindernotfallversorgung von ausgewiesenen Experten in praxisorientierter Form nach aktuellem Wissensstand zusammengefasst.

Demirakca zeigt die Differenzialdiagnosen und die wichtigsten Therapiemaßnahmen bei einer der häufigsten Einsatzindikationen bei Kindern auf: dem respiratorischen Notfall. Aufgrund der meist obstruktiven Problematik wird hier der Schwerpunkt auf eine frühzeitige und aus- reichend dosierte Inhalationstherapie gelegt.

Löllgen und Szabo beleuchten die verschiedenen Schockformen im Kindesalter. Hierbei wird deutlich, dass die frühzeitige klinische Erkennung des Schocks im Kindesalter sehr schwierig ist und entgegen der Erwachsenenmedizin die Blutdruckmessung unzuverlässig ist, weshalb der Beurteilung der kapillären Füllungszeit große Bedeutung zukommt. Hier wird u. a. das frühzeitige Legen eines intraossären Zugangs thematisiert und die wichtige Initialtherapie mit Flüssigkeitsboli von bis zu $3 \times 20 \mathrm{ml} / \mathrm{kgKG}$ hervorgehoben. Außerdem wird auch die initiale Katecholamintherapie eines Kinds im Schock abgehandelt.

Landsleitner und Keil beschreiben das Vorgehen bei einem Verbrennungstrauma. Dieser Beitrag legt den Schwerpunkt auf eine adäquate Schmerztherapie im Kindesalter und zeigt die einfache Möglichkeit einer intranasalen Medikamentenapplikation, die gerade in dieser Notfallsituation zu einer deutlichen Verbesserung der Versorgungsqualität geführt hat, auf. In sehr praxisorientierter kasuistikbasierter Form werden alltagstaugliche Empfehlungen zusammengefasst.

Heimberg et al. stellen wichtige Fehlerursachen beim Kindernotfall zusammen und berichten über aktuelle Entwicklungen zur Vermeidung oder Beherrschung dieser typischen Probleme bei der Versorgung der kritisch kranken Kinder. Ein Schwerpunkt des Beitrags stellt dabei die Früherkennung von lebensbedrohlichen Zuständen im Kindesalter dar. Dies scheint aufgrund des Pathomechanismus des kindlichen Herz-Kreislauf-Stillstands geradezu von eklatanter Bedeutung für die Verbesserung des Outcomes von Kindernotfällen zu sein. Außerdem 
gehen die Autoren auf die teilweise unzureichende Ausrüstung für Kindernotfälle ein. Die Notwendigkeit einer Standardisierung und einer Abstimmung des präund innerklinischen Bereichs wird hier thematisiert.

Kollege Strauß widmet sich dem Problem der gewichtsadaptierten Medikamentenapplikation und bewertet die verfügbaren Hilfsmittel zur Dosierung von Medikamenten im Kindesalter mit dem Ziel, die Fehlerrate bei der Dosierung von Medikamenten im Notfall zu verringern. Gerade die korrekte Dosierung bei einem sehr breiten Altersspektrum stellt Nichtpädiater immer wieder vor große Herausforderungen.

Die vorliegende Ausgabe der Zeitschrift Medizinische Klinik - Intenisvmedizin und Notfallmedizin stellt somit einen praxisorientierten und einprägsamen Überblick über wichtige Themen der pädiatrischen Notfallmedizin dar. Es bleibt zu hoffen, dass die in diesen Übersichtsarbeiten publizierten Standards zu einer weiteren Verbesserung der Notfallversorgung im Kindesalter beitragen.

\section{Qhiver finsel}

Dr. Oliver Heinzel, Tübingen

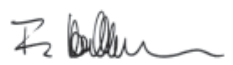

Dr. Florian Hoffmann, München

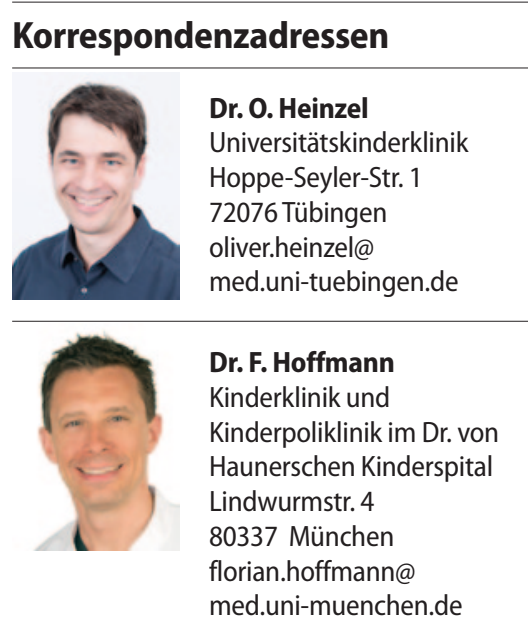

\section{Einhaltung ethischer Richtlinien}

Interessenkonflikt. O. Heinzel und F. Hoffmann erhalten Honorar als Simulationstrainer für den Verein PAEDSIM e. V. - Teamtraining für Kindernotfälle sowie Vortragshonorare der Firma MedUpdate $\mathrm{GmbH}$.

\section{Anmerkung der Redaktion}

Der Beitrag von J. Strauß kann leider nicht im Rahmen dieses Leitthemas erscheinen.

\section{Hans-Georg-Mertens-Preis 2016}

Bewerbungsfrist: 31. August 2015, Dotation: 5.000 Euro

Der H. G. Mertens-Preis wird gemeinsam von der Deutschen Gesellschaft für Neurologie e.V. (DGN) und der Deutschen Gesellschaft für Neurolntensiv- und Notfallmedizin e.V. (DGNI) ausgeschrieben.

Der Preis wird seit 2003 für innovative, therapierelevante Forschung in der Neurologischen Intensivmedizin und der Allgemeinen Neurologie verliehen.

Die Auszeichnung wird während der Eröffnungsveranstaltung auf der Arbeitstagung der DGNI vom 28.-30. Januar 2016 in Berlin in Form einer Urkunde verliehen und ist mit einer Geldprämie von 5.000 Euro verbunden.

\section{Preiskuratorium}

Das Preiskuratorium setzt sich aus den jeweiligen 1. Vorsitzenden der DGN und DGNI und je zwei weiteren Vertretern beider Fachgesellschaften zusammen.

Für die Deutsche Gesellschaft für Neurologie e.V.:

- 1. Vorsitzender: Prof. Dr. Ralf Gold (Bochum)

- Prof. Dr. Martin Grond (Siegen)

- Dr. Gunther Carl (Kitzingen)

Für die Deutsche Gesellschaft für Neurolntensiv- und Notfallmedizin e.V.:

- 1. Vorsitzender: Prof. Dr. Jürgen Meixensberger (Leipzig)

- Prof. Dr. Wolfgang Müllges (Würzburg)

- Prof. Dr. Georg Gahn (Karlsruhe)

\section{Bewerbung}

Die Bewerbungsunterlagen sollen ein Bewerbungsschreiben, die drei wichtigsten Arbeiten, das Schriftenverzeichnis, den Lebenslauf des Bewerbers sowie eine kurze Darstellung des Inhaltes der besonderen wissenschaftlichen Leistungen (Publikationen, Habilitationsschrift) umfassen. Sie sind bevorzugt als PDF per E-Mail bis zum 31. August 2015 an die Geschäftsstelle der Deutschen Gesellschaft für Neurologie e.V., Reinhardtstraße 27 C, 10117 Berlin, E-Mail: info@dgn.org zu richten. 